\title{
Interactive teaching methods of optoelectronics for enhancing engagement of under-represented groups
}

Pouya Dianat

Pouya Dianat, "Interactive teaching methods of optoelectronics for enhancing engagement of under-represented groups," Proc. SPIE 11143, Fifteenth Conference on Education and Training in Optics and Photonics: ETOP 2019, 111430K (2 July 2019); doi: 10.1117/12.2523869 


\title{
Interactive teaching methods of optoelectronics for enhancing engagement of under-represented groups
}

\author{
Pouya Dianat \\ Electrical and Computer Engineering Department, Drexel University, 3141 Chestnut St, \\ Philadelphia PA, 19104, USA
}

\begin{abstract}
An engaging teaching method provides an equal learning opportunity for students on the often-arduous concepts in optoelectronics, which enhances involvement of minorities during a lecture session. Experiment-oriented instruction of topics combined with a dynamic and dialogue-based delivery of the lectures has showed increased participation from the class. Such experiments must be designed based on commonly used optoelectronic devices i.e. LEDs, to provoke interest among the learners. Furthermore, the students have to investigate non-trivial characteristics of such devices, e.g. measuring emission spectrum with a hand-held spectrometer, in groups of two-three. These would ensure a balanced learning opportunity among all the students.
\end{abstract}

Keywords: Interactive teaching, Optoelectronics, Photonics, STEM, Under-represented groups

\section{INTRODUCTION}

Among various fields in Science, Technology, Engineering, and Mathematics (STEM), there exists a large gender gap in computer science, engineering and physics; women have generally demonstrated a preference to pursue degrees in biology, chemistry, and mathematics. ${ }^{1}$ As highlighted by Cheryan $\mathrm{et} \mathrm{al}^{1}$ the main reasons accounting for this disparity with physics and computer sciences field are: (a) masculine cultures that signal a lower sense of belonging to women than men, (b) a lack of sufficient early experience with computer science, engineering, and physics, and (c) gender gaps in self-efficacy. Furthermore, same reasons may explain the lower rate of persistency in the STEM field majors for under-represented groups. ${ }^{2}$ Additionally, racial prejudice and stereotyping have been shown to affect various ethnicities differently, ${ }^{3}$ as explained by Zou and Cheryan, ${ }^{4}$ based on racial perceptions of African-, Asian-, and Latino-Americans. While fundamental steps are required to be taken at early stages in a broad educational system of a country, in order to rectify the class culture and enhance exposure for under-represented groups in a university setting, and specifically for highly specialized fields of photonics and optoelectronics, a course instructor plays a critical role as the moderator and authoritative figure. ${ }^{5}$ An instructor may take an interactive approach, ${ }^{6,7}$ as a means to engage minorities, through placing them in leadership roles, imposing inquiries and questions, and incentivizing participation.

The author of this article has developed, and utilized interactive techniques, ${ }^{8}$ with the purpose of enhancing minority engagement throughout the courses taught to juniors and seniors focused on optoelectronics and at Drexel University. As an example, in a lecture designed to teach the relationship between wavelength and energy of photons and the band-gap of various semiconductor materials, an interacting and inclusive environment was established by having a judgment-free dialogue with students based on asking questions on the topic and inquiring students' opinions or conducting surveys on the subject during the lecture. Upon concluding the lecture, the students were asked to form groups of two to three, and were asked to obtain the emission spectrum of LEDs with different colors with a hand held spectrometer. This step provided an equal opportunity for all the students to further interact with the subject matter and have learning re-enforcements through immediate observation of newly learned material. This method is easy to apply, and have shown enhanced engagement among all the students and specifically minorities to interact with the topic as well as with their peers. Further details on these are elaborated in the following sections.

Further author information: (Send correspondence to Pouya Dianat)

E-mail: dianat@drexel.edu, Telephone: 12157968315

Fifteenth Conference on Education and Training in Optics and Photonics: ETOP 2019, edited by

Anne-Sophie Poulin-Girard, Joseph A. Shaw, Proc. of SPIE Vol. 11143, 111430K · (c) 2019

SPIE, ICO, IEEE, OSA·CCC code: 0277-786X/19/\$18 · doi: 10.1117/12.2523869

Proc. of SPIE Vol. 11143 111430K-1 


\section{A METHOD TO ENHANCE ENGAGEMENT IN TEACHING PHOTONICS}

It is well established that interactive-engagement (IE) methods of education enhance problem-solving abilities for students in physics courses in all levels, from high-school to university, beyond that obtained in traditional practice. $^{6,9}$ As a subset of physics, photonic-based course have to follow similar strategies for a better return on the time invested by the students. This, in particular, is of more importance as the topics covered in photonics may be more intimidating for students due to the amount of math and concept rigor. Importantly, the utilization of IE methods places the instructor in a unique position as the class moderator to oversee interactions, engage under-represented groups, and enhance class involvement.

In photonics education and particularly for undergraduates, the learning process typically includes: a) presentation of the concepts through a lecture, followed by b) lab experimentation as reinforcement. The author has constructed lectures on photonics and optoelectronics topic on an inquiry-based method, through which the attention span and engagement of students were increased. As indicated in Fig. 1, the flow diagram followed for an IE photonics lecture includes:

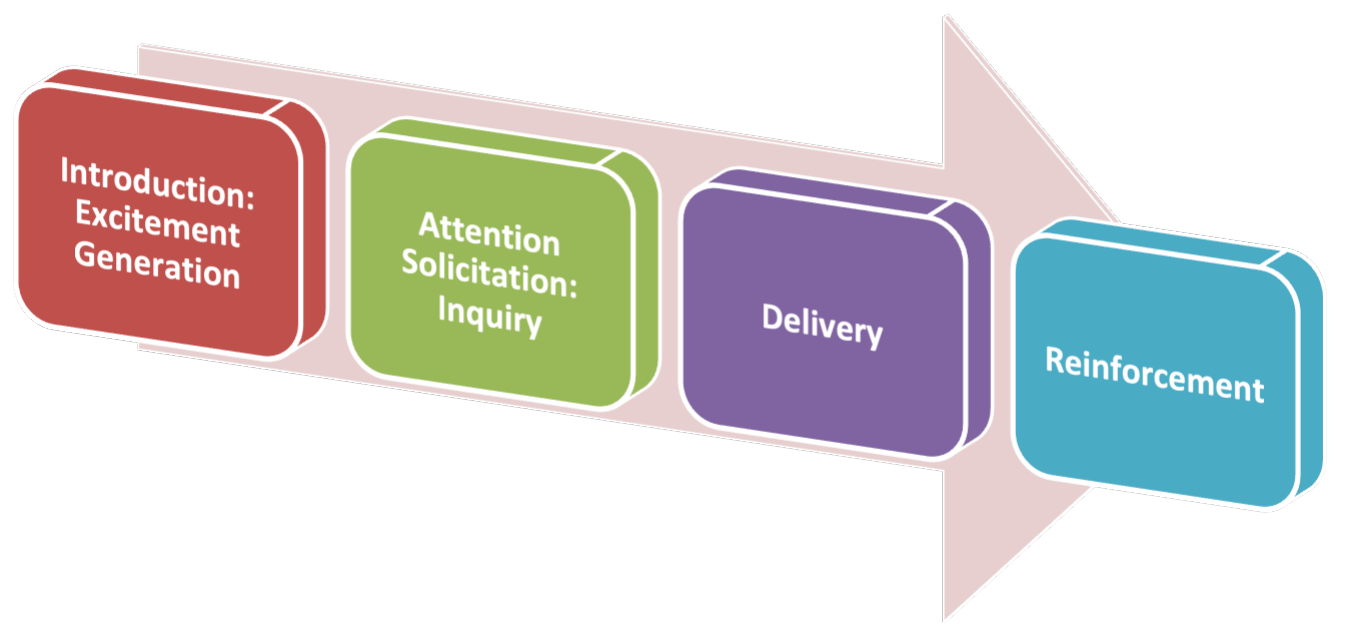

Figure 1. Steps pursued for an interactive and inclusive delivery of topics in optoelectronics.

1. Topic Introduction: This step is critical to generate excitement among learners. This starts from surveying the class by asking about their general knowledge on the topic of interest. For instance the students are asked about their familiarity with LEDs as a more-and-more prominent light source in their daily lives. General questions about the meaning of colors and their relationship to wavelengths are asked and the general knowledge of the class is surveyed and if necessary, the relevant physics topics are reviewed. It ensures that the whole class will be at the same level of background knowledge for the topic to be covered. It is essential in generating excitement and remove stress of learning new material for the whole class and to provide equal learning opportunity.

2. Attention Solicitation: Upon introduction to a topic, a dialogue is initiated by the instructor with the students by asking them questions and inquiries based on their daily life experiences. For instance, the following questions are typically asked from the students to attract their attention to the significance of light spectrum: "Why is human eye sensitive to the visible wavelength, while a snake's eye receives infrared?, What is the difference between sun light and fluorescent light source as both are sources of white light?, Why is the latter not considered a high quality light?" The students readily do not have a prepared answers 
to these questions, although they deal with them on a daily basis. Therefore, it intrigues them to find the answer.

Importantly, these questions should not be targeted to the whole class; instead, they should be asked from individuals to start a conversation. This opportunity may be used to build upon and involve minorities in the topic of discussion by inviting them to participate.

3. Delivery: Once the attention of the class is secure, the answers are delivered to the students through a logical build-up by referring to the underlying math for the phenomenon of discussion. This ensures that the lecture is purposeful and signifies the physical modeling behind it. Fig. 2 shows one possible representation of the answer to previous inquiries.

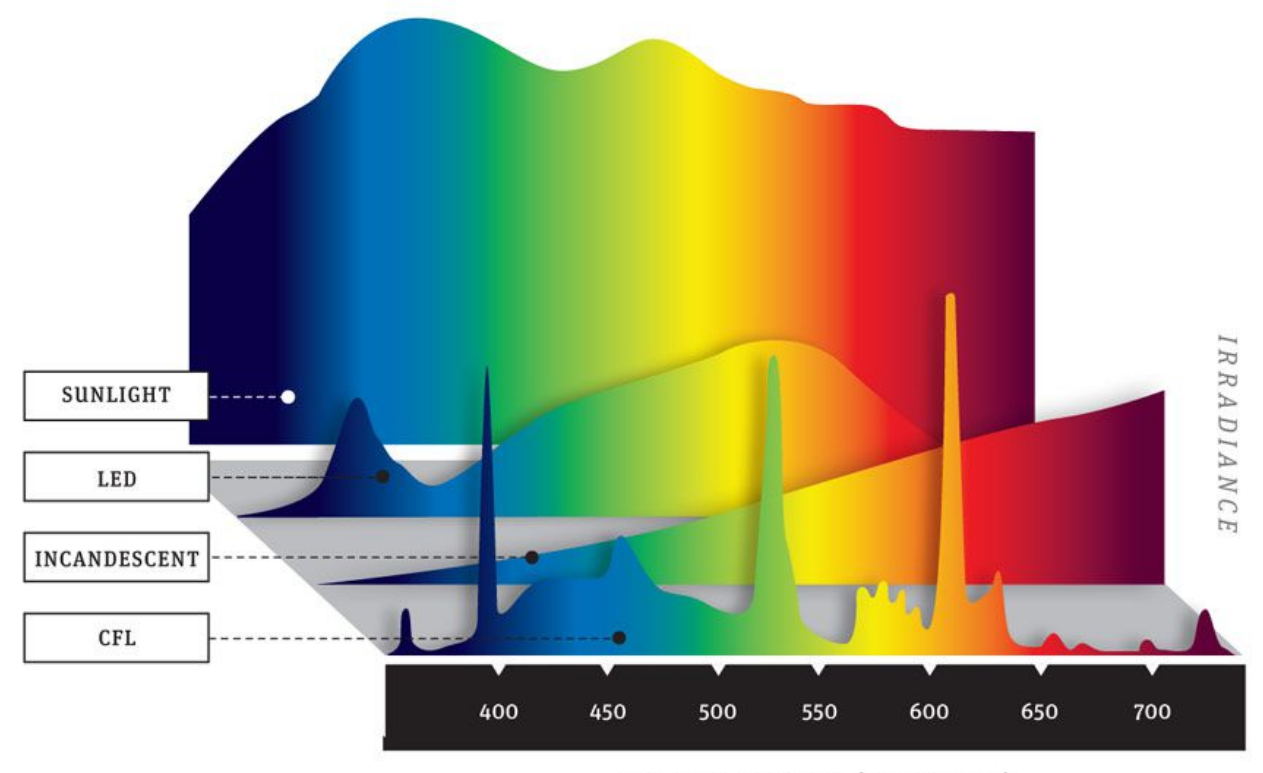

WAVELENGTH (nanometers)

Figure 2. Spectrum curves for various light sources used to motivate students on light-wavelength relationship. Picture courtesy of ${ }^{12}$

4. Reinforcement: Finally, new inquiries are made based on the newly gained knowledge. The students are asked to form small groups and collaborate on finding the answer and presenting their findings to the rest of the class in a debate format. In our example of spectrum of LED, the students are asked to relate the energy of a photon to its wavelength and based on that justify why an LED with shorter wavelength will typically require a larger bias voltage to start emitting light. This step serves as a bridge to experimental work in the lab.

\section{INCLUSIVITY ENHANCEMENT THROUGH LAB EXPERIMENTS}

Of particular interest is utilization of lab experiments as a means to increase leadership and involvement of minorities in a given topic. I have always asked the students to conduct the lab experiments as part of a group of 2-3 with a preference on a larger group size. As the instructor, I have utilized the following strategies to craft a class culture with increased inclusion and participation in the course:

- Mandatory participation: It is necessary to ensure that the learners all will gain a minimum required knowledge from the course material. A mandatory credited participation will serve this purpose. This could be achieved by asking each student to present a project, explain the solution to homework problems 
to the whole class, or merely participate in the dialogues with the instructor. This will not only help the instructor gauge the progress of the students, but also paves the way for equalizing learning opportunity for all groups. Importantly, the instructor has to make sure that this is a judgement-free practice. Students have to be reassured that it is their participation that matters, and not whether they know the right answer. Incorporation of peer instruction may also be essential in furtherance of influence of participation. ${ }^{10}$

- Bonus Projects: Experiments should be designed to not be cumbersome, otherwise it would result in boredom and unnecessary stress with limited educational value. For instance for our light source spectrum experiments, the students should be provided with an easy-to-use device such as an Ocean Optics hand held spectrometer (Fig. ??), to attain the spectrum. The students may be provided with the fundamentals of operation of the device, however, it should not be focus of the experiment. Rather, a bonus project may be assigned for those who are interested to go above and beyond the required course work. My experience indicates that under-represented students are more inclined in completing these bonus project. This observation may be in line with the results of a recent study on gender-equality paradox STEM education, ${ }^{11}$ where it is suggested that "life-quality pressures in less gender-equal countries promote girls' and women's engagement with STEM subjects."

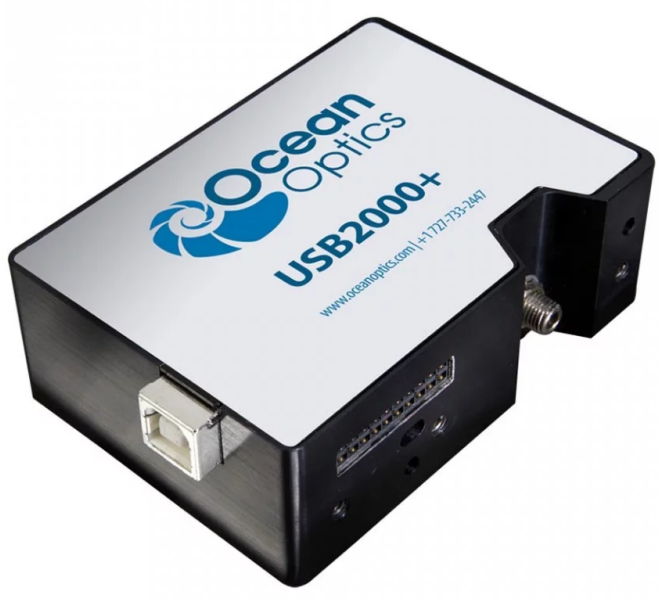

Figure 3. A hand held Ocean Optics USB2000+ spectrometer used for conducting the light source experiment.

- Leadership assignment: Commonly, in a group of three, a student becomes an implied leader, while another student will be a mediator, between the leader and the third member. Through instructor intervention, these roles may be switched and controlled. For instance in the LED experiment, the implied leader is typically the student who communicates the results with the instructor, and asks questions regarding the validity of the spectrum data. By establishing a group dialogue targeted at the other groups members the leadership role may be switched and modified. This provides an opportunity to implicitly provide leadership opportunity for minorities in a group.

- Individuality recognition: Finally, as human beings everyone is mostly receptive to their names; therefore, as an instructor I always call my students by their first names and give them the deserved recognition to the whole audience during a lab or lecture. This is more crucial and should be practiced more often about minority students, to ensure they develop a sense of belonging to the class. This simple step, also plays a significant role in setting class culture and promotes involvement and equality among everyone.

These key points are also summarize in Fig. 4. 


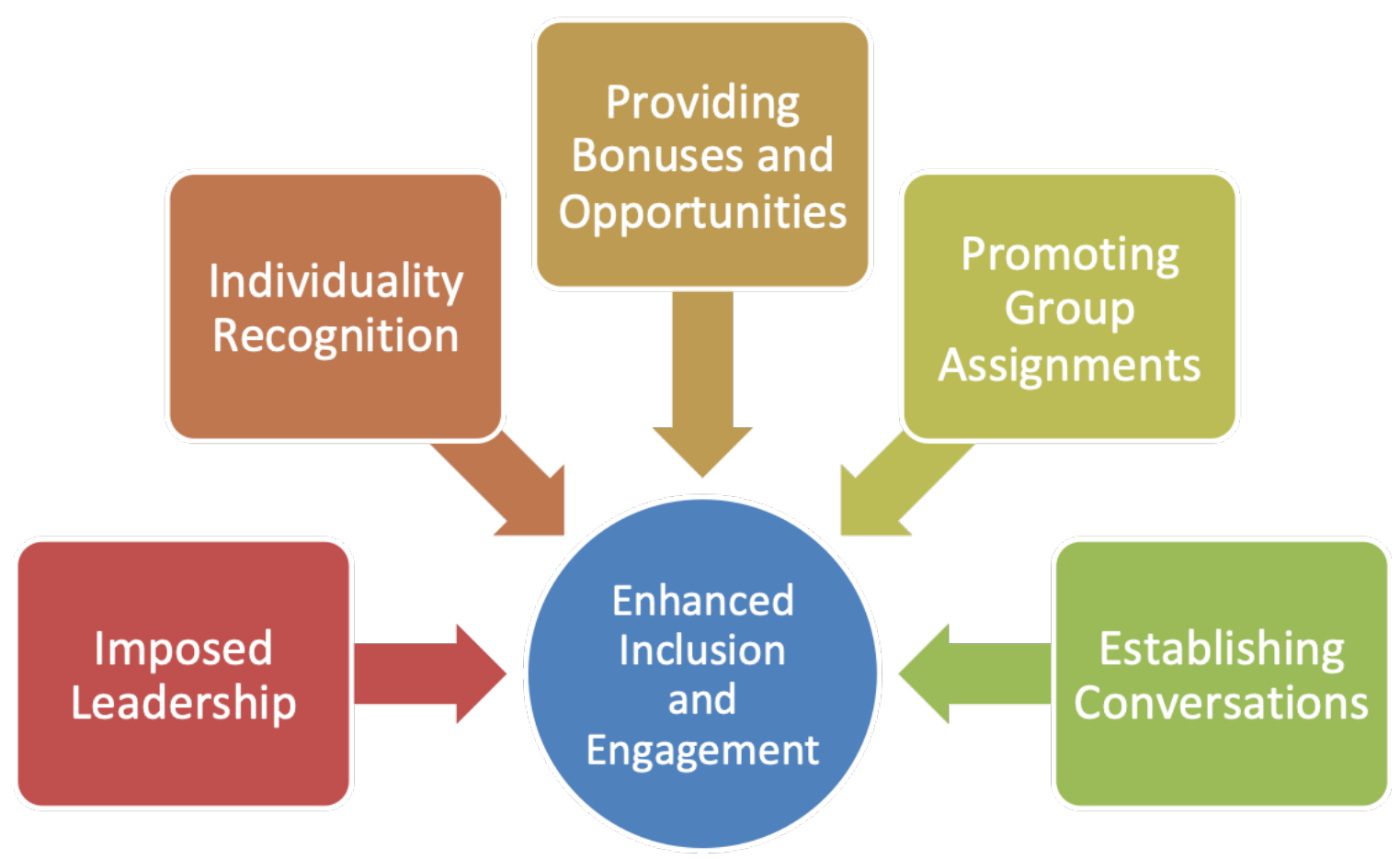

Figure 4. Steps pursued for an interactive delivery of lectures on optoelectronics.

\section{CONCLUSION}

Practical Interactive-Engagement methods and tips for teaching photonics to undergraduate students were discussed that provided equal learning opportunity for students and enhanced involvement of minorities during a lecture session. Experiment-oriented instruction of topics combined with a dynamic and dialogue-based delivery showed increased participation from the class. As an example, experiments to obtain spectrum of LEDs was presented as a way to provoke interest among the learners. Group activity among students ensured involvement of minorities in the learning process and their assumption of leadership positions.

\section{REFERENCES}

[1] Cheryan, S., Ziegler, S. A., Montoya, A. K., and Jiang, L., "Why are some stem fields more gender balanced than others?," Psychological Bulletin 143(1), 1 (2017).

[2] Griffith, A. L., "Persistence of women and minorities in stem field majors: Is it the school that matters?," Economics of Education Review 29(6), 911-922 (2010).

[3] Chen, J. M., Pauker, K., Gaither, S. E., Hamilton, D. L., and Sherman, J. W., "Black+ white= not white: A minority bias in categorizations of black-white multiracials," Journal of Experimental Social Psychology $\mathbf{7 8}$, 43-54 (2018).

[4] Zou, L. X. and Cheryan, S., "Two axes of subordination: A new model of racial position.," Journal of personality and social psychology $\mathbf{1 1 2}(5), 696$ (2017).

[5] McGrady, P. B. and Reynolds, J. R., "Racial mismatch in the classroom: Beyond black-white differences," Sociology of Education 86(1), 3-17 (2013).

[6] Hake, R. R., "Interactive-engagement versus traditional methods: A six-thousand-student survey of mechanics test data for introductory physics courses," American journal of Physics 66(1), 64-74 (1998). 
[7] Jensen, J. L., Kummer, T. A., and Godoy, P. D. d. M., "Improvements from a flipped classroom may simply be the fruits of active learning," CBE? Life Sciences Education 14(1), ar5 (2015).

[8] Prince, M., "Does active learning work? a review of the research," Journal of engineering education 93(3), 223-231 (2004).

[9] Karamustafaoglu, O., "Active learning strategies in physics teaching.," Online Submission 1(1), 27-50 (2009).

[10] Crouch, C. H. and Mazur, E., "Peer instruction: Ten years of experience and results," American journal of physics 69(9), 970-977 (2001).

[11] Stoet, G. and Geary, D. C., "The gender-equality paradox in science, technology, engineering, and mathematics education," Psychological science 29(4), 581-593 (2018).

[12] Herrman, J., "Ultimate Light Bulb Test: Incandescent vs. Compact Fluorescent vs. LED." Pupular Mechanics, 20 September 2011 https://www.popularmechanics.com/technology/gadgets/reviews/g164/ incandescent-vs-compact-fluorescent-vs-led-ultimate-light-bulb-test/. (Accessed: 01 May 2019). 